“Abnormal vertebral patterns in genetically heterogeneous deceased fetuses and neonates: evidence of selection against variations"

Figure count: 6 and 1 supplementary figure

7 Ms. Pauline C. SCHUT, MD ${ }^{a^{*}}$, Erwin BROSENS, PhD ${ }^{b}$, Frietson GALIS, PhD ${ }^{c}$, Clara M. A. TEN

$12{ }^{a}$ Department of Obstetrics and Gynecology, Division of Obstetrics and Fetal Medicine, Erasmus

13 MC, University Medical Center Rotterdam, Rotterdam, The Netherlands

$14{ }^{b}$ Department of Clinical Genetics, Erasmus MC, University Medical Center Rotterdam,

15 Rotterdam, The Netherlands 
17 d Department of Radiology, Division of Paediatric Radiology, Erasmus MC, University Medical

18 Center Rotterdam, Rotterdam, The Netherlands

19 e Department of Paediatric Surgery, Erasmus MC, Erasmus University Medical Center Sophia

20 Children's Hospital, Rotterdam, The Netherlands

21

$22 *$ Corresponding author:

23 Pauline $\mathrm{C}$. Schut

24 Erasmus MC, University Medical Center Rotterdam, Department of Obstetrics and Gynecology,

25 Division of Obstetrics and Fetal Medicine, Rotterdam, The Netherlands

26 Room Na-1608

27 Postbus 2040

283000 CA Rotterdam, The Netherlands

29 E-mail: p.schut@erasmusmc.nl

30 Work phone number: 0031107033917

31 Cell phone number: 0031613191240

32 Fax: 0031107035826 


\section{Author contributions:}

35 T.C., A.E., F.G., C.B., E.B., A.K. and P.S. designed the study. I.B. en P.S. collected patient data. P.S.

36 reviewed the radiographs. E.B. and M.S. performed the genetic analyses. P.S. and E.B. wrote

37 the manuscript. All authors revised the manuscript critically and all authors approved the final version of the manuscript.

Competing interests: The authors declare no competing interests.

41 Funding sources: None.

42

43

44

45

46

47 
Abstract

Objective: To assess the vertebral pattern in a cohort of deceased fetuses and neonates, and to

51 study the possible impact of DNA Copy Number Variations (CNVs) in coding regions and/or

52 disturbing enhancers on the development of the vertebral pattern.

53 Method: Radiographs of 445 fetuses and infants, deceased between 2009 and 2015, were

54 assessed. Terminations of pregnancies, stillbirths and neonatal deaths were included. Patients

55 were excluded if the vertebral pattern could not be determined. Copy number profiles of 265

56 patients were determined using single nucleotide polymorphism array.

57 Results: $274 / 374$ patients (73.3\%) had an abnormal vertebral pattern. Cervical ribs were

present in $188 / 374(50.3 \%)$ and were significantly more common in stillbirths $(69 / 128(53.9 \%))$ and terminations of pregnancies $(101 / 188(53.7 \%))$, compared to live births $(18 / 58,31.0 \%, p=$ patterning. Conclusion: The presence of an abnormal vertebral pattern, particularly in the cervical region, could be a sign of disruption at critical, highly interactive and conserved stages of embryogenesis. The vertebral pattern might provide valuable information regarding fetal and neonatal outcome. CNV analyses did not identify a mutual genetic cause for the occurrence of 
vertebral patterning abnormalities, indicating genetic heterogeneity.

67

68 Key words: cervical ribs, congenital anomalies, evolutionary selection, homeotic

69

transformation, Copy Number Variants, rib number, supernumerary ribs, vertebral pattern.

70

71 Introduction

72 The human vertebral column normally consists of 7 cervical, 12 thoracic, 5 lumbar, 5 sacral and

73 3-4 coccygeal vertebrae. Only the thoracic vertebrae are rib-bearing. Deviations from this

74 vertebral pattern rarely occur in healthy individuals, particularly in the cervical region. ${ }^{1}$

75 However, variations in cervical patterning, including (rudimentary) cervical ribs, have been

76 described in specific populations. ${ }^{2,3}$ In the presence of cervical ribs, a partial or full posterior

77 homeotic transformation of the seventh cervical vertebra has occurred, because the vertebra

78 has features of a rib-bearing thoracic vertebra. This results in a change in the number of true

79 cervical and thoracic vertebrae and consequently to a shift of the cervicothoracic junction. ${ }^{4}$

80 It has been hypothesized that the lack of variation in cervical vertebral patterning is the result

81 of developmental constraints or evolutionary selection against changes. ${ }^{5-8}$ The low prevalence

82 of cervical ribs in healthy pediatric or adult populations, compared to the high prevalence in 
83 deceased fetuses and neonates, supports this hypothesis. ${ }^{7,9} \mathrm{~A}$ high prevalence of abnormalities

84 in vertebral patterning is also found in children with specific pediatric malignancies. ${ }^{10-13}$

85 Furthermore, Galis et al. ${ }^{7}$ concluded that the majority of individuals with cervical ribs, are not

87 life-threatening, cervical ribs could be regarded as markers of disadvantageous embryonic 
101 The aims of the current study were to examine the prevalence of abnormal vertebral patterns

102 in deceased fetuses and neonates and to determine whether an abnormal vertebral pattern is

103 associated with (specific) structural and/or chromosomal anomalies in this population. This

104 study also aimed to gain insight into the possible impact of DNA Copy Number Variations

105 (CNVs) in coding regions and/or disturbing enhancers on the development of the pattern of the

106 vertebral column.

107

108 Methods

109 Study population

110 The cohort consisted of fetuses and infants, younger than one year old, deceased between

1112009 and 2015 in the Erasmus University Medical Center Sophia Children's Hospital, of whom a

112 babygram, autopsy report and/or SNP Array was available. Spontaneous intrauterine fetal

113 demise and medically indicated terminations of pregnancies were included. Medical

114 information about mothers, fetuses and neonates were retrieved from electronic medical

115 records.

116 Babygrams were made routinely if autopsy was performed. Autopsies were performed

117 according to the national guidelines. ${ }^{23}$ Congenital anomalies were categorized according to the

118 European Registry of Congenital Anomalies and Twins (Eurocat) classification system. ${ }^{24}$ If 
119 autopsy had not been performed, the presence of malformations was based on the report of

120 the prenatal advanced ultrasound scan, pre- or postnatal radiographic investigations or post-

121 mortem external inspection. If none of these investigations had been requested, or the results

122 were inconclusive (e.g. due to maceration), the presence of congenital anomalies was

123 categorized as non-available.

124 Radiographs were made both ventrally and laterally (5.6-12.6mAs, 40-50 kV, Philips Optimus

125 ZBM3/NZR91, Philips Medical Systems, Eindhoven, the Netherlands). Radiographs performed at

126 other hospitals were requested. All radiographs were assessed by one reviewer, who was

127 blinded for the autopsy results and the results of genetic investigations. Rudimentary ribs and

128 the vertebral pattern were defined following ten Broek et al. ${ }^{6}$ : if a rib on the most cranial or

129 most caudal thoracic vertebra had a length of less than half of the rib of the adjacent thoracic

130 vertebra, it was considered rudimentary. Rudimentary cervical ribs were scored if the length of

131 the transverse processes of the seventh cervical vertebra was more than the transverse process

132 of the first thoracic vertebra, but less than half of the first thoracic rib. If the rib was longer than

133 half of the first thoracic rib, it was considered a (complete) cervical rib. Deviations from the

134 normal vertebral pattern were classified as more severe when cranially located vertebral

135 regions and multiple vertebral regions were involved (figure 1). 
136 A subset of thirty randomly chosen radiographs was assessed twice by the same reviewer and

137 by a second reviewer, to determine the intraobserver and interobserver variability.

138 The study was performed according to the Dutch law on clinical trials in the Netherlands

139 (WMO). Under this law the study does not require ethical approval or informed consent. This

140 was confirmed by the institutional ethics committee (Medical Research Ethics Committee

141 Erasmus MC, MEC-2014-098).

142 Analysis of copy number variation

143 DNA was isolated from material that was collected in patients opting for invasive prenatal or

144 postnatal diagnostic tests. We determined the CNV profiles in all coding and non-coding regions

145 of patients ( $n=265)$ using methods and analysis settings previously described. ${ }^{25}$ CNV profiles

146 were inspected visually in Biodiscovery Nexus CN8.0. (Biodiscovery Inc., Hawthorne, CA, USA).

147 Rare CNVs were classified as described in table 1 and inspected for overlap with candidate

148 genes, such as HOX genes and genes previously reported to be associated with vertebral

149 anomalies in VACTER-L patients. ${ }^{26,27}$ A more detailed description of this analysis can be found

150 in the supplementary methods.

\section{Statistical analysis}

152 Statistical analysis was performed using SPSS Statistics (IBM Corp. Released 2013. IBM SPSS

153 Statistics for Windows, Version 21.0. Armonk, NY: IBM Corp.). Determination of differences 
154 between groups were calculated using independent sample t-tests for continuous data and chi-

155 square test and Fisher's exact test for categorical data. Descriptive statistics were used to

156 evaluate outcome parameters. A p-value <0.05 was considered statistically significant. Kappa's

157 test was performed in order to determine the interobserver and intraobserver reliability. Kappa

158 values between 0.61 and 0.80 were considered to be substantial and kappa values between

1590.81 and 1.00 were considered almost perfect. ${ }^{28}$

160 Results

161 The study population consisted of 374 fetuses and 71 neonates. In 71 fetuses and neonates

162 (16.0\%), the vertebral pattern could not be reliably assessed by radiography. This was due to

163 inadequate positioning of the fetus or neonate, overprojection of the maxilla, clavicles or

164 umbilical cord clamp, or low quality of the babygram. Excluding these 71 patients, 316 fetuses

165 and 58 neonates were available for analysis of the pattern of the vertebral column. No

166 statistically significant differences were found between the included and excluded patients in

167 gestational age, presence or type of congenital anomaly and pregnancy outcome (table S1). Of

168 the 374 included fetuses and neonates, 188 (50.3\%) pregnancies were terminated because of

169 medical reasons, mostly (suspected) fetal structural, chromosomal or other genetic anomalies

170 ( $N=180 / 188,95.7 \%)$. Other medical indications for pregnancy terminations were severe fetal

171 growth restriction $(\mathrm{N}=1,0.5 \%)$, preterm premature rupture of membranes $(\mathrm{N}=3,1.6 \%)$ or poor 
172 maternal condition in pregnancies with early onset severe pre-eclampsia or HELLP syndrome

$173(\mathrm{~N}=4,2.1 \%)$. The second largest group consisted of miscarriages or intrauterine fetal demises

$174(128 / 374,34.2 \%)$. The majority of the 58 neonatal deaths was related to structural anomalies

175 (43/58, 74.1\%). Most other causes of neonatal death were associated with prematurity (table

176 S2).

177 An overview of maternal, fetal and neonatal characteristics is provided in table 2. The inclusion

178 of multiple pregnancies and more than one pregnancy per mother resulted in a total number of

179366 included mothers. Autopsy was performed in the majority of patients (N=305, 81.6\%). In 35

180 of the 69 patients in whom no autopsy was performed, an advanced ultrasound examination

181 was carried out (9.4\%). The presence of structural abnormalities could not be ascertained in 40

182 patients. This included 31 still births, 6 terminations of pregnancy and 3 live births. In 33 of

183 these patients, neither autopsy nor advanced ultrasound examination had been performed. In 7

184 patients, the results of autopsy or advanced ultrasound examination were inconclusive.

185 Structural anomalies were present in a large proportion of the group (256/334, 76.6\%) and the

186 prevalence was highest in the subgroup of pregnancy terminations (173/182, 95.1\%), compared

187 to $43 / 55(78.2 \%)$ in live births and $40 / 97$ (41.2\%) in stillbirths. In more than half of the patients

188 with a structural anomaly, more than one organ system was affected $(146 / 256,57.0 \%)$. The

189 most frequently affected organ systems were the cardiovascular $(N=90)$, nervous $(N=85)$, 
craniofacial $(N=82)$, limbs $(N=71)$ and urogenital $(N=66)$ system (figure 2$)$.

191

A regular vertebral pattern was identified in approximately one quarter of patients (100/374,

$26.7 \%$, figure 3). Of the 274 patients (73.3\%) with an abnormal vertebral pattern, the

$68.1 \%)$; of the unilateral cervical ribs, most were left sided $(N=37 / 60,61.7 \%)$. Figure 4 shows a babygram of a fetus with rudimentary twelfth thoracic ribs and rudimentary cervical ribs, and a babygram of a neonate with a regular vertebral pattern.

The distribution of the vertebral pattern categorized according to structural abnormality is 70.8\%), abnormalities involving the digestive system $(28 / 46,60.9 \%)$ and limb defects (43/71, 60.6\%). The most disturbed vertebral pattern (CT_TL_LS) was frequently seen in patients with 
abnormalities.

209

After subdivision of the study population in stillbirths, live births and terminations of

pregnancies, it became clear that the proportion of fetuses and neonates with a regular

211 vertebral pattern was significantly higher in live births $(25 / 58,43.1 \%)$, compared to stillbirths

$212(29 / 128,22.7 \%)$ and terminations of pregnancies $(46 / 188,24.5 \%, p=0.009)$. Cervical ribs were

213 significantly more common in stillbirths (69/128 (53.9\%) and terminations of pregnancies

214 (101/188 (53.7\%), compared to live births $(18 / 58,31.0 \%, p=0.006)$. The distribution of the

215 vertebral pattern in these subgroups is shown in figure 6. The prevalence of cervical ribs did not

216 differ significantly between fetuses and neonates with and without structural anomalies in the

217 total group $\left(126 / 256\right.$ versus $\left.40 / 78, X^{2}(1)=0.10, p=0.80\right)$, nor in the subgroups of live births

$218(13 / 43$ versus $4 / 12, p=1.0)$, stillbirths $\left(21 / 40\right.$ versus $\left.32 / 57, X^{2}(1)=0.13, p=0.72\right)$, or

219 terminations of pregnancies $(92 / 173$, versus $4 / 9, p=0.74)$. The 4 live births with cervical ribs,

220 but without structural abnormalities died because of a subgaleal hemorrhage, sepsis, asphyxia

221 and uterine rupture, respectively. Autopsy was performed in all of these neonates. The

222 asphyctic neonate had mild dysmorphic features, but a normal karyotype. The pregnancy in

223 which a uterine rupture occurred was complicated by polyhydramnios and macrosomia.

224 Maternal diabetes was ruled out. The macrosomic neonate had mild dysmorphic features and

225 was suspected of having an overgrowth syndrome, but additional DNA-testing did not reveal a 
genetic mutation. Chromosomal or genetic analyses were not performed in the remaining 2

227 neonates.

228 No statistically significant difference was found in the prevalence of cervical ribs between males

229 and females $\left(109 / 208\right.$ versus $\left.79 / 166, X^{2}(1)=0.86, p=0.36\right)$. When the group was categorized

230 according to the number of affected organ systems, the proportion of fetuses and neonates

231 with a disturbed vertebral pattern involving all vertebral boundaries (CT_TL_LS) was highest in

232 the subgroup with four or more affected organ systems (fig S1).

233 Gestational age at birth $(M=23.5$ weeks, $S D=6.2$, versus $M=23.7$ weeks, $S D=6.3, p=0.73)$,

234 maternal smoking $(p=0.39)$, assisted conception $(p=0.47)$, use of folic acid $(p=0.77)$ and

235 maternal $B M I(M=24.8, S D=5.3$, versus $M=24.7, S D=4.7, p=0.91)$ were not significantly

236 different between fetuses and neonates with and without cervical ribs.

237 The intra-observer reliability was almost perfect for determination of the number of thoracic

238 ribs (Kappa $=0.84)$ and substantial for assessment of the presence of cervical ribs (Kappa $=$

239 0.78). The interrater reliability was substantial for both determination of the number of

240 thoracic ribs $($ Kappa $=0.77)$ and cervical ribs $($ Kappa $=0.74)$.

241 Karyotype was available in 221 (59.1\%) patients. Aneuploidies were detected in 15 (6.8\%)

242 patients; $10(66.7 \%)$ of these had cervical ribs. Microarray tests were performed in 265 (70.9\%)

243 of patients. Neither karyotype nor array was available in 54 patients (14.4\%). In 31 patients 
$244(31 / 265,11.7 \%)$ microarray showed an abnormality. Cervical ribs were present in 20/31 (64.5\%)

245 patients with an abnormal microarray result. The presence of cervical ribs in patients with a

246 pathogenic or (likely) deleterious CNV was 50.0\% (6/12) and 57.1\% (4/7), respectively. These

247 data are summarized by subgroup in table S3.

248 CN profiling

249 The prevalence of large (likely) deleterious CNV (table 2) and aneuploidies was high. However,

250 there were no recurrent or overlapping rare CNVs. Some of the rare CNVs have overlap with

251 dominant disease genes (see table S4). There was no overlap with candidate genes (e.g. the

252 HOX gene cluster) Pathway enrichment analysis of genes affected by a rare CNV did not provide

253 evidence of enrichment of relevant pathways.

\section{Discussion}

255 An abnormal pattern of the vertebral column, including the presence of cervical ribs, was

256 frequently found in deceased fetuses and neonates. The prevalence was noticeably higher in

257 this population, compared to living children or adults who are not known to have structural,

258 chromosomal or genetic abnormalities, as reported in the literature. ${ }^{9}$ These findings are in line

259 with the study of ten Broek et al., who included a similar study population. ${ }^{6}$ The differences in

260 vertebral pattern between subgroups categorized into affected organ system were less obvious

261 compared to the study of ten Broek et al. ${ }^{6}$, which could be due to the smaller number of 
262 included patients in our study. The high frequency of the most severely disturbed vertebral

263 pattern in the subgroups with ventral body wall defects, craniofacial and skeletal malformations

264 was confirmed. The co-occurrence of an abnormal vertebral pattern and these abnormalities

265 could be explained by the close spatial relationship and the intense interaction of signaling

266 pathways between the embryonic precursors of these organ systems and the vertebral column

267 in early embryogenesis. ${ }^{29-31}$ For instance, signaling from the somites influences the migration of

268 neural crest cells, from which the craniofacial skeleton is derived, whereas somites themselves

269 give rise to the vertebrae and ribs, ventral body wall skeleton, abdominal muscles, skeletal

270 muscles and cartilage. Because of the intense interactions between developmental processes

271 during somitogenesis, this period is considered an extremely vulnerable period. ${ }^{32,33}$

272 Consequently, disruptions in vertebral patterning would often be accompanied by disruptions

273 of other developmental processes. As expected, the most severely disturbed vertebral pattern

274 was most frequent in patients with more than four affected organ systems. This might be due

275 to a prolonged disturbance of developmental processes.

276 Unexpectedly, the prevalence of cervical ribs was not significantly different between fetuses

277 and neonates with and without structural abnormalities. This is in contrast to ten Broek et al. ${ }^{6}$,

278 but has also been observed by Furtado et al. ${ }^{14}$, who suggested that incomplete penetrance of

279 genetic events leading to the occurrence of both abnormal vertebral patterning and fetal death, 
280 could result in a milder phenotype without structural abnormalities. Thus the presence of

281 cervical ribs might not only be associated with the presence of structural abnormalities, but

282 also with the occurrence of intrauterine fetal demise itself, even in the absence of structural

283 abnormalities detected by autopsy. The significantly higher prevalence of cervical ribs in the

284 subpopulation of stillbirths compared to live births is in line with this theory. The relatively high

285 prevalence of cervical ribs within the subpopulation of deceased neonates without proven

286 structural abnormalities $(4 / 11,36.4 \%)$ remains unexplained, however. The disappearance of

287 cervical ribs later in fetal life or childhood has also been considered, but seems less plausible, as

288 cervical ribs are frequently encountered in specific (adult) patient groups. ${ }^{11,12,34}$ In addition, no

289 differences between the presence of cervical ribs in fetuses and infants was found by Galis et

290 al. $^{7}$ and the prevalence of cervical ribs was not substantially lower in adults compared to

291 children in the general population. ${ }^{9}$

292 Cervical ribs were detected in the majority of patients with an aneuploidy or abnormal

293 microarray result. The reported prevalence of cervical ribs in aneuploidies ranges between 12.5

294 and $100 \%{ }^{14,35}$ Studies reporting on cervical ribs in populations with microarray abnormalities

295 are lacking. The number of patients with similar chromosomal or genetic abnormalities in this

296 cohort was insufficient to enable the detection of statistically significant associations between

297 cervical ribs and specific chromosomal or genetic abnormalities. 
Overlapping abnormalities involving HOX-genes or other candidate genes were not identified

within this cohort. This does not completely rule out a common genetic basis for the

abnormalities of the vertebral pattern, because the presence of structural DNA variations, such

as point mutations, are not detected by SNP array and CNV analyses. However, both the

included in this study population proved to have different chromosomal or genetic

stillbirths (those with cervical ribs) had a common underlying genetic basis.

The occurrence of similar segmentation anomalies in patients with various structural,

313 the cause of the disruption itself. ${ }^{38-44}$ Abnormalities in vertebral patterning, such as cervical

314 ribs, could thus be regarded as a sign of abnormal embryonic development, irrespective of the 
regular pattern of the vertebral column in this study population of deceased fetuses and neonates, underwrites the hypothesized selection against variations in the conserved process

of vertebral patterning.

319 These findings indicate that assessment of the vertebral pattern could be of added value in

320 determination of fetal and neonatal outcome. Prenatal assessment of the vertebral pattern,

321 including the detection of rudimentary cervical ribs, using (3-dimensional) ultrasound seems

322 feasible. ${ }^{45-50}$

323 Strengths of this study are the large study population and the good intraobserver and

324 interobserver reliability for the assessment of the vertebral pattern and cervical ribs on

325 radiographs. Limitations are the small sizes of the subgroups with specific structural,

326 chromosomal or genetic abnormalities and the fact that autopsy had not been performed in all

327 patients. Although the presence of a healthy control group was lacking, literature regarding the

328 prevalence of cervical ribs in healthy populations was available for comparison.

\section{Conclusion}

330 The presence of abnormalities in the pattern of the vertebral column, particularly in the cervical

331 region, could be regarded as a sign of disruption at critical, interactive and conserved stages of

332 early embryonic development. The absence of rare recurrent CNVs, and the presence of similar 
334 abnormalities are an indication of the genetic heterogeneity that appears to underlie the

335 development of an abnormal vertebral pattern. Assessment of the vertebral pattern could

336 provide valuable information regarding fetal and neonatal outcome.

337 Further studies investigating the feasibility and value of prenatal (3-dimensional) ultrasound

338 assessment of the number of vertebrae and ribs are warranted. Whole exome sequencing on

339 subjects with (isolated) vertebral patterning abnormalities might provide insight into the

340 presumably heterogeneous genetic causes of these patterning defects.

\section{Acknowledgements}

342 The authors thank Ms. S.C. Husen ${ }^{a}$, for the assessment of the subset of radiographs for

343 determination of the interobserver reliability. No funding source or compensation.

344 a Department of Obstetrics and Gynecology, Division of Obstetrics and Fetal Medicine, Erasmus

345 MC, University Medical Center Rotterdam, Rotterdam, The Netherlands

346 Data availability

347 The datasets generated during and/or analyzed during the current study are available from

348 the corresponding author on reasonable request. 


\section{References}

352

3531 Galis, F. Why do almost all mammals have seven cervical vertebrae? Developmental

354

355 constraints, Hox genes, and cancer. J Exp Zool 285, 19-26 (1999). Brewin, J., Hill, M. \& Ellis, H. The prevalence of cervical ribs in a London population. Clin Anat 22, 331-336 (2009). Etter, M. Osseous abnormalities of the thoracic cage seen in forty thousand consecutive chest photoroentgenograms. Am J Roentgenol 51, 359-363 (1944). Bots, J. et al. Analysis of cervical ribs in a series of human fetuses. J Anat 219, 403-409 (2011).

Narita, Y. \& Kuratani, S. Evolution of the vertebral formulae in mammals: a perspective on developmental constraints. J exp zool B Mol Dev Evol 304, 91-106 (2005). Ten Broek, C. M. et al. Evo-Devo of the Human Vertebral Column: On Homeotic Transformations, Pathologies and Prenatal Selection. Evol Biol 39, 456-471 (2012). Galis, F. et al. Extreme selection in humans against homeotic transformations of cervical vertebrae. Evolution 60, 2643-2654 (2006). Varela-Lasheras, I. et al. Breaking evolutionary and pleiotropic constraints in mammals: On sloths, manatees and homeotic mutations. Evodevo 2, 11 (2011). Schut P. C. et al. Adverse Fetal and Neonatal Outcome and an Abnormal Vertebral Pattern: A Systematic Review. Obstetrical \& Gynecological Survey 71, 741-750 (2016). Loder, R. T., Huffman, G., Toney, E., Wurtz, L. D. \& Fallon, R. Abnormal rib number in childhood malignancy: Implications for the scoliosis surgeon. Spine 32, 904-910 (2007). anomalies and childhood cancers. Br J Cancer 105, 1392-1395 (2011). childhood cancer patients. Eur J Med Genet 48, 113-129 (2005).

13 Schumacher, R., Mai, A. \& Gutjahr, P. Association of rib anomalies and malignancy in childhood. Eur J Pediatr 151, 432-434 (1992).

14 Furtado, L. V., Thaker, H. M., Erickson, L. K., Shirts, B. H. \& Opitz, J. M. Cervical ribs are more prevalent in stillborn fetuses than in live-born infants and are strongly associated with fetal aneuploidy. Pediatr Dev Pathol 14, 431-437 (2011).

15 Lappin, T. R., Grier, D. G., Thompson, A. \& Halliday, H. L. HOX genes: seductive science, mysterious mechanisms. Ulster Med J 75, 23-31 (2006). Mallo, M., Wellik, D. M. \& Deschamps, J. Hox genes and regional patterning of the vertebrate body plan. Dev Biol 344, 7-15 (2010).

17 Quinonez, S. C. \& Innis, J. W. Human HOX gene disorders. Mol Genet Metab 111, 4-15 (2014). cervical vertebrae in Hoxa-4 mutant mice. Proc Natl Acad Sci U S A 91, 12644-12648 (1994). 
39320 Manley, N. R. \& Capecchi, M. R. Hox group 3 paralogs regulate the development and 394 migration of the thymus, thyroid, and parathyroid glands. Dev Biol 195, 1-15 (1998).

39521 Anbazhagan, R., Raman V. . Homeobox genes: molecular link between congenital anomalies and cancer. Eur J Cancer 33, 635-637 (1997).

22 van der Lugt, N. M. et al. Posterior transformation, neurological abnormalities, and severe hematopoietic defects in mice with a targeted deletion of the bmi-1 protooncogene. Genes Dev 8, 757-769 (1994).

40023 https://www.nvk.nl/Nieuws/Dossiers/NODO. (September 12th 2018). EUROCAT. Coding of EUROCAT Subgroups of Congenital Anomalies. Chapter 3.3, Guide 1.4 (2012).

40325 Brosens, E. et al. Copy number variations in 375 patients with oesophageal atresia and/or tracheoesophageal fistula. Eur J Hum Genet 24, 1715-1723 (2016).

26 Chen, Y. et al. The genetic landscape and clinical implications of vertebral anomalies in VACTERL association. J Med Genet 53, 431-437, doi:jmedgenet-2015-103554 [pii]10.1136/jmedgenet-2015-103554 (2016).

27 Solomon, B. D. et al. Clinical geneticists' views of VACTERL/VATER association. Am J Med Genet A 158A, 3087-3100 (2012). Landis, J. R. \& Koch, G. G. The measurement of observer agreement for categorical data. Biometrics 33, 159-174 (1977).

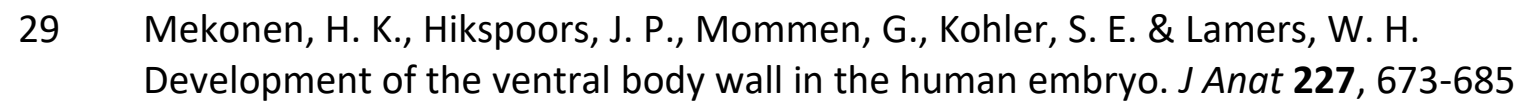
(2015). spine in unoperated infants with cleft lip and palate. Cleft Palate Craniofac J 43, 513-518 (2006). Morphology. Int J Dent 2010, 295728 (2010).

32 Lubinsky, M. Blastogenetic associations: General considerations. Am J Med Genet $A$ 167A, 2589-2593 (2015).

33 Galis, F. \& Metz, J. A. Testing the vulnerability of the phylotypic stage: on modularity and evolutionary conservation. J Exp Zool 291, 195-204, doi:10.1002/jez.1069 (2001).

34 Weber, A. E. \& Criado, E. Relevance of bone anomalies in patients with thoracic outlet syndrome. Ann Vasc Surg 28, 924-932 (2014).

Schut, P. C. et al. Increased prevalence of abnormal vertebral patterning in fetuses and neonates with trisomy 21. J Matern Fetal Neonatal Med, 1-7 (2018). Martinez-Frias, M. L. Segmentation anomalies of the vertebras and ribs: one expression of the primary developmental field. Am J Med Genet A 128A, 127-131, doi:10.1002/ajmg.a.30016 (2004). Giampietro, P. F. et al. Clinical, genetic and environmental factors associated with congenital vertebral malformations. Mol Syndromol 4, 94-105 (2013).

38 JG, W. Methods for administering agents and detecting malformations in experimental animals. In: Wilson, J.G. and Warkany, J., Eds., Teratology: Principals and Techniques University of Chicago Press, Chicago, 262-277 (1965).

39 Lu, C. C., Matsumoto, N. \& lijima, S. Teratogenic effects of nickel chloride on embryonic 
mice and its transfer to embryonic mice. Teratology 19, 137-142, doi:10.1002/tera.1420190202 (1979).

40 Lu, F. Basic toxicology. Fundamentals, target organs and risk assessment. . Bristol, PA: Taylor and Francis. (1991).

41 Sadler, T. W. Effects of maternal diabetes on early embryogenesis: II. Hyperglycemiainduced exencephaly. Teratology 21, 349-356, doi:10.1002/tera.1420210311 (1980).

42 De Sesso JM, H. S. Principles underlying developmental toxicity. In: Fan AM, Chang LW, editors. Toxicology and risk assessment principles, methods, and applications. , 37-56 (1996).

43 Opitz, J. M. The developmental field concept. Am J Med Genet 21, 1-11, doi:10.1002/ajmg.1320210102 (1985).

44 Lubinsky, M. Associations in clinical genetics with a comment on the paper by Evans et al. on tracheal agenesis. AM J MED GENET 21, 35-38 (1985).

45 Khodair, S. A., Hassanen, O.A. Abnormalities of fetal rib number and associated fetal anomalies using three dimensional ultrasonography. The Egyptian Journal of Radiology and Nuclear Medicine 45, 689-694 (2014).

46 Hershkovitz, R. Prenatal diagnosis of isolated abnormal number of ribs. Ultrasound Obstet Gynecol 32, 506-509 (2008).

47 Gindes, L., Benoit, B., Pretorius, D. H. \& Achiron, R. Abnormal number of fetal ribs on 3dimensional ultrasonography: Associated anomalies and outcomes in 75 fetuses. $J$ Ultrasound Med 27, 1263-1271 (2008).

48 T. Esser, P. R., N. Sarioglu, K.D. Kalache. . Three-dimensional ultrasonographic demonstration of agenesis of the 12th rib in a fetus with trisomy 21. Ultrasound in Obstetrics \& Gynecology 27, 712-715 (2006).

49 A. Dall'Asta, G. P., C.C. Lees. Crystal Vue technique for imaging fetal spine and ribs. Ultrasound in Obstetrics \& Gynecology 47, 383-384 (2016).

50 Schut, P.C., Verdijk, R. M., Joosten, M. \& Eggink, A. J. Prenatal diagnosis of cervical ribs by three-dimensional ultrasound in a foetus with a herniated Dandy-Walker cyst. $B M J$ Case Rep 11, doi:11/1/e225381 [pii]10.1136/bcr-2018-225381 (2018). 


\section{Figures}

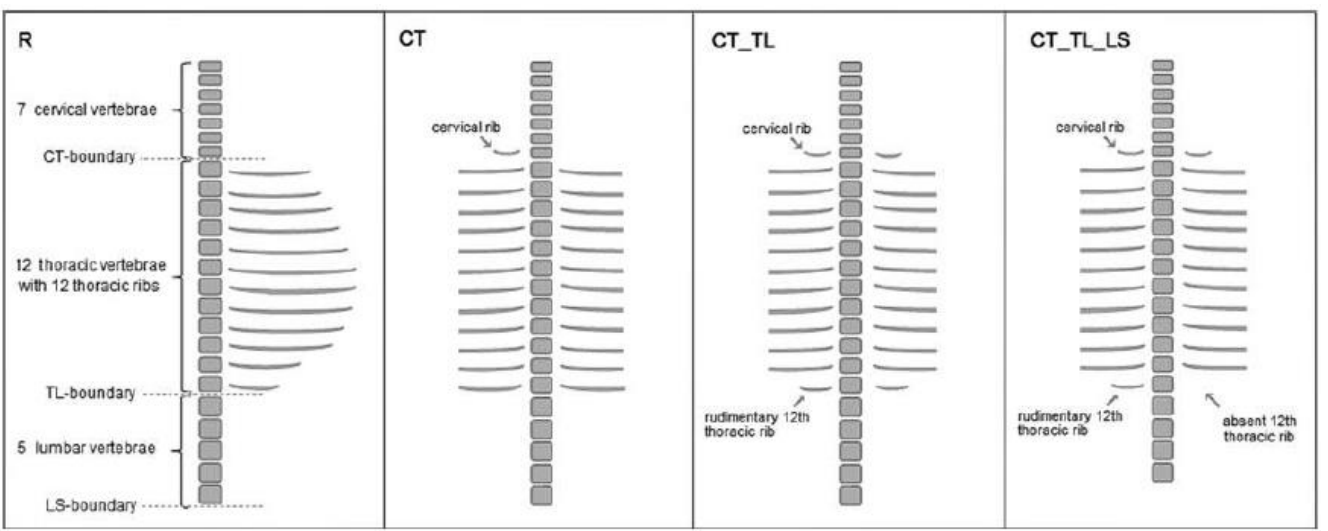

477 Figure 1. Overview of different vertebral columns and number on severity scale. From left to right: $\mathrm{R}=$ Regular pattern, severity scale value $0 ; \mathrm{CT}=$ shift at the cervicothoracic boundary, severity scale value $4 ; \mathrm{CT}_{-} \mathrm{TL}=$ shift at the cervicothoracic and thoracolumbar boundary, severity scale value 6; CT_TL_LS= shift at cervicothoracic, thoracolumbar and lumbosacral boundary, severity scale value 7 .

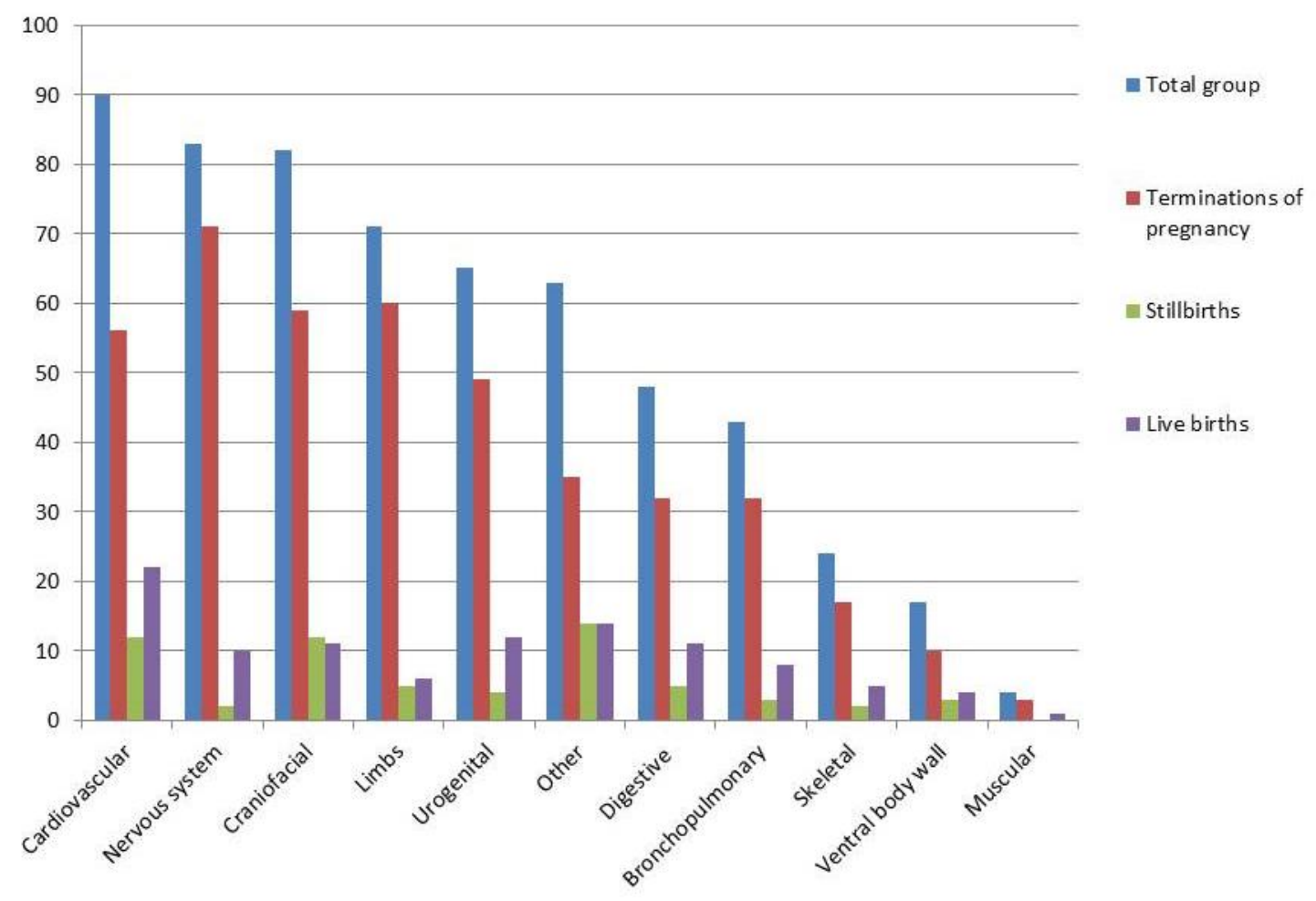

Figure 2. The distribution of structural abnormalities in the total study population and subgroups of live births, stillbirths and terminations of pregnancy.

$485 \mathrm{CT}=$ shift at the cervicothoracic boundary, $\mathrm{CT}_{-} \mathrm{TL}=$ shift at the cervicothoracic and 486 thoracolumbar boundary, CT_LS= shift at cervicothoracic and lumbosacral boundary, TL_LS= 
487 shift at thoracolumbar and lumbosacral boundary, CT_TL_LS= shift at cervicothoracic, 488 thoracolumbar and lumbosacral boundary.

489

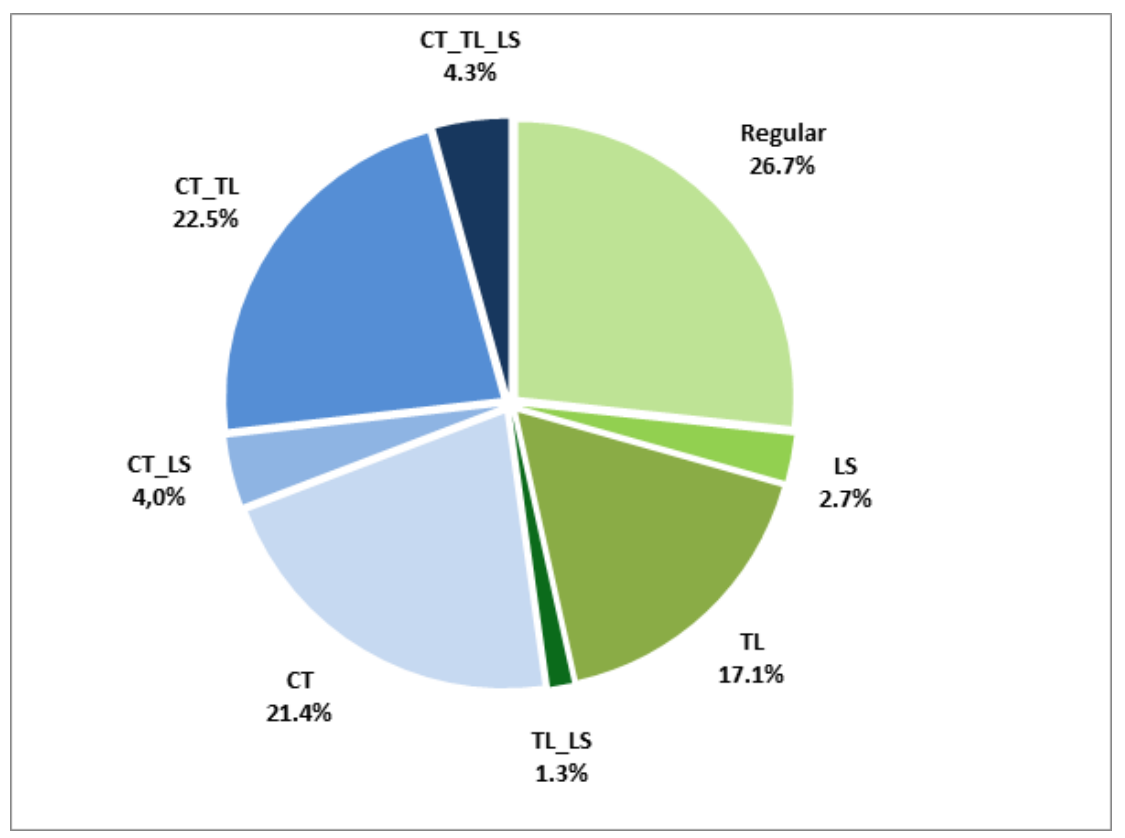

491 Figure 3. The distribution of the different patterns of the vertebral column in the total study 492 population $(\mathrm{N}=374)$

$493 \mathrm{CT}=$ shift at the cervicothoracic boundary, $\mathrm{CT}_{-} \mathrm{TL}=$ shift at the cervicothoracic and

494 thoracolumbar boundary, CT_LS= shift at cervicothoracic and lumbosacral boundary, TL_LS= 495 shift at thoracolumbar and lumbosacral boundary, CT_TL_LS= shift at cervicothoracic, 496 thoracolumbar and lumbosacral boundary. 


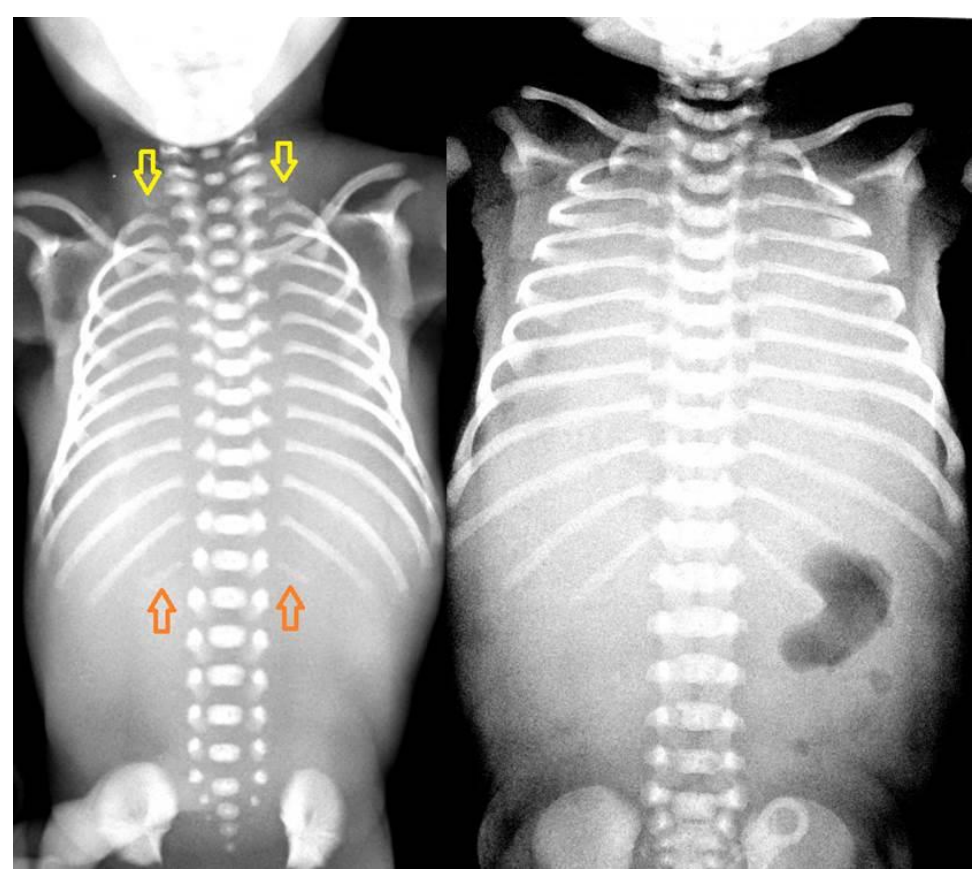

499 Figure 4. Babygram of a fetus at 20.1 weeks' gestation (left) and a deceased term neonate

500 (right). The babygram on the left shows rudimentary 12th thoracic ribs (orange arrows) and the 501 presence of rudimentary cervical ribs (yellow arrows); the babygram on the right shows 12 502 thoracic rib pairs and the absence of cervical ribs. 


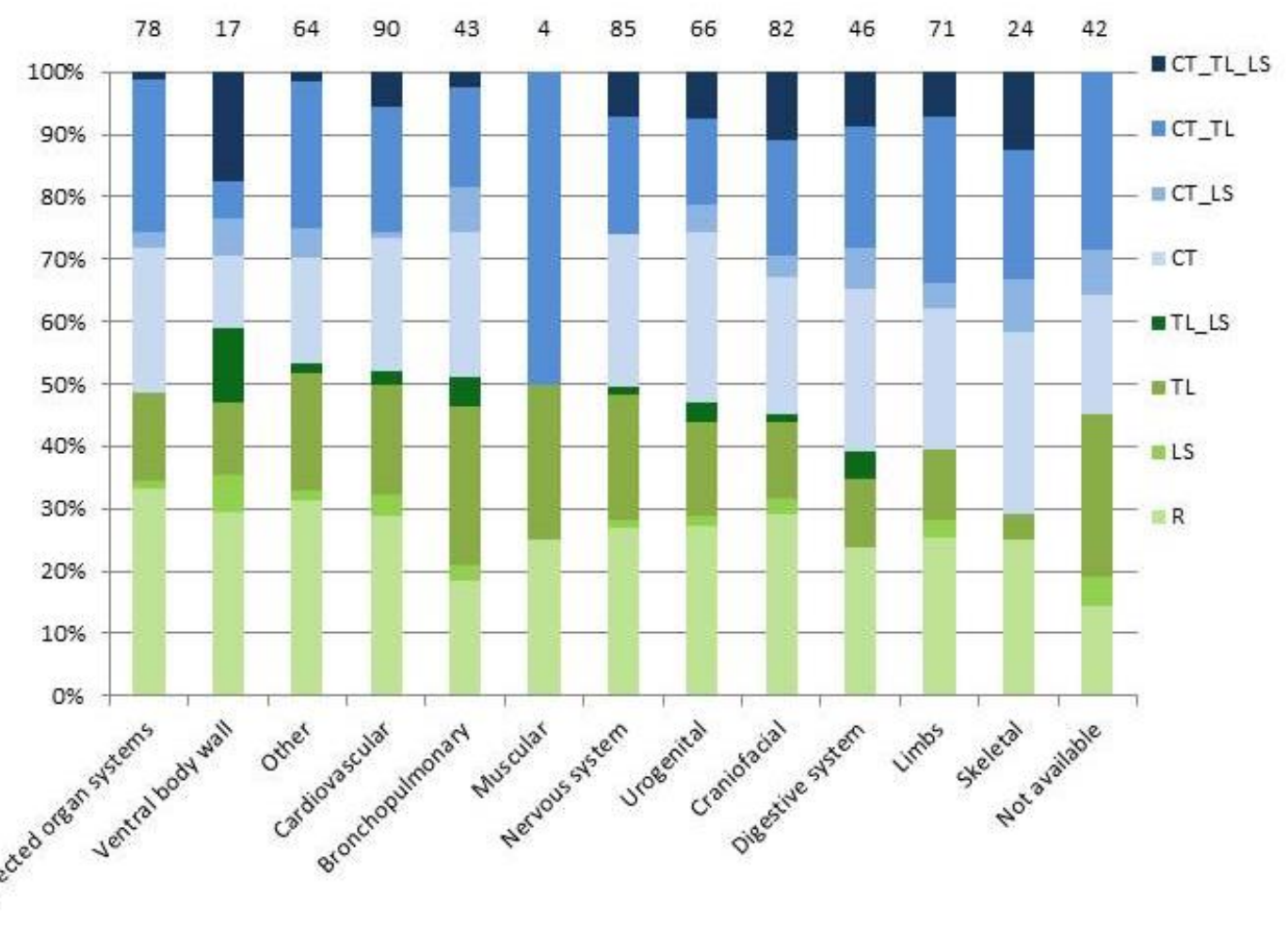

505 Figure 5. The pattern of the vertebral column in fetuses and neonates categorized according to 506 the presence and type of structural anomaly. The total number of cases in each group is 507 depicted above the bars.

$508 \mathrm{R}=$ Regular pattern, $\mathrm{CT}=$ shift at the cervicothoracic boundary, $\mathrm{CT}$ _ $\mathrm{TL}=$ shift at the 509 cervicothoracic and thoracolumbar boundary, CT_LS= shift at cervicothoracic and lumbosacral 510 boundary, TL_LS= shift at thoracolumbar and lumbosacral boundary, CT_TL_LS= shift at 511 cervicothoracic, thoracolumbar and lumbosacral boundary. 


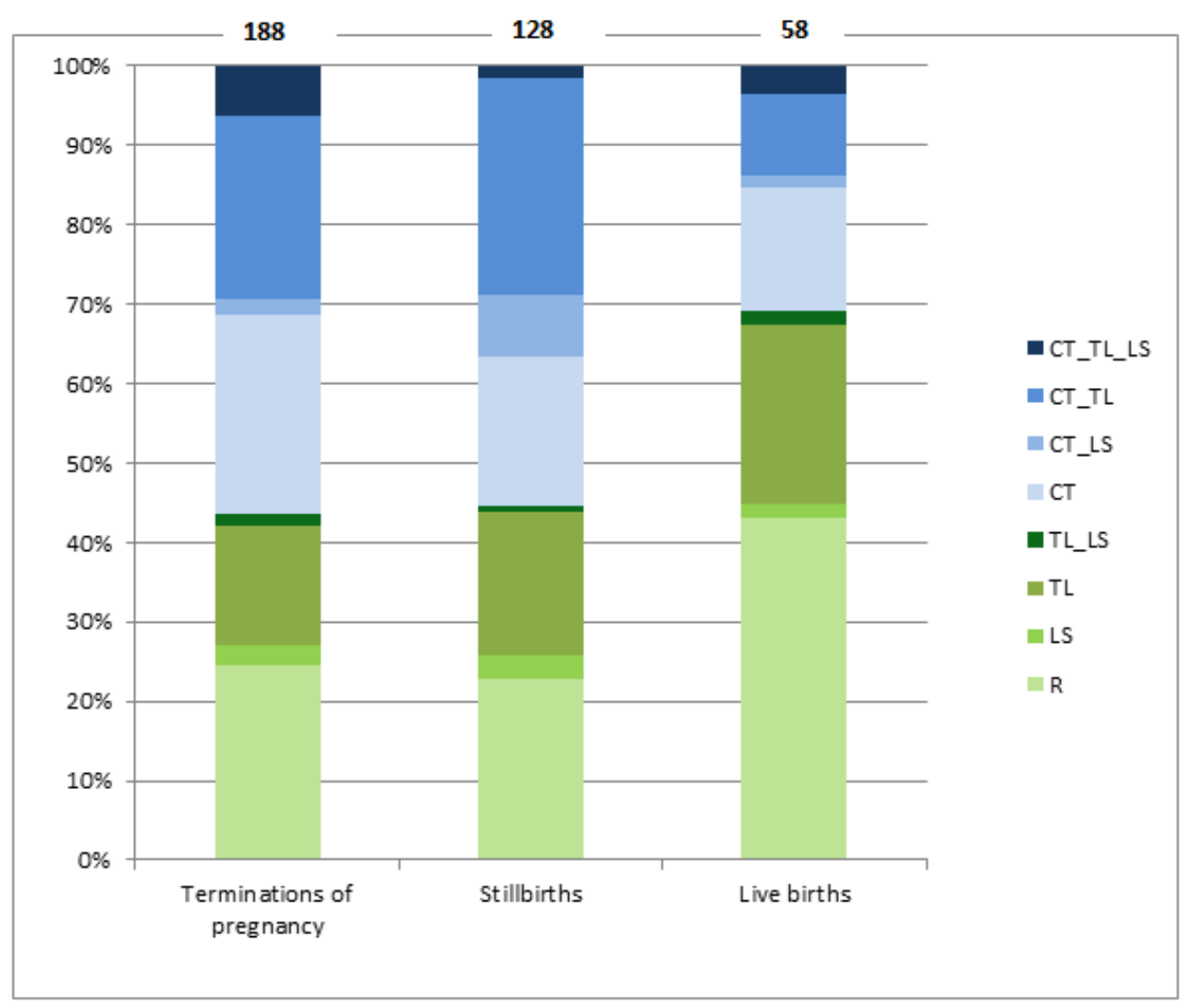

514 Figure 6. The distribution of the vertebral pattern in terminations of pregnancies, still births and 515 live births. The total number of cases is depicted above the bars.

$516 \mathrm{R}=$ Regular pattern, $\mathrm{CT}=$ shift at the cervicothoracic boundary, $\mathrm{CT}$ _ $\mathrm{TL}=$ shift at the

517 cervicothoracic and thoracolumbar boundary, CT_LS= shift at cervicothoracic and lumbosacral

518 boundary, TL_LS= shift at thoracolumbar and lumbosacral boundary, CT_TL_LS= shift at

519 cervicothoracic, thoracolumbar and lumbosacral boundary.

520

521

522

523

524

525

526

527 
531

\begin{tabular}{|c|c|c|c|}
\hline Mothers & N (366) (\%) & Fetuses/neonates & N (374) (\%) \\
\hline Age (years) & $31.0(15-44)$ & \multirow{3}{*}{$\begin{array}{l}\text { Sex } \\
\text { Male } \\
\text { Female }\end{array}$} & \multirow{3}{*}{$\begin{array}{l}208(55.6) \\
166(44.4) \\
\end{array}$} \\
\hline $\mathrm{BMI}\left(\mathrm{kg} / \mathrm{m}^{2}\right)$ & $23.6(16.4-44.6)$ & & \\
\hline Missing & 73 & & \\
\hline Smoking & & Pregnancy outcome & \\
\hline Yes & $44(12.0)$ & Miscarriages and stillbirths & $128(34.2)$ \\
\hline Quit & $15(4.1)$ & Live births & $58(15.5)$ \\
\hline No & $259(70.8)$ & Neonatal death $<1 w k$ & 45 (77.6) \\
\hline Unknown & $48(13.1)$ & Termination of pregnancy & $188(50.3)$ \\
\hline Parity & & Gestational age at birth (weeks) & $22.7(11.9-41.3)$ \\
\hline Primigravida & $125(34.2)$ & Live births & $30.9(22.3-41.3)$ \\
\hline Multigravida & $239(65.3)$ & Miscarriages and stillbirths & $22.6(13.6-40.3)$ \\
\hline Unknown & $2(0.5)$ & Terminations of pregnancy & $22.0(11.9-33.6)$ \\
\hline Folic Acid & & Multiple pregnancies & \\
\hline Yes, preconception & $140(38.3)$ & Single & $347(92.8)$ \\
\hline Yes, postconception & $118(32.2)$ & Twins & $26(7.0)$ \\
\hline No & $38(10.4)$ & Triplets & $1(0.3)$ \\
\hline Unknown & $70(19.2)$ & & \\
\hline Conception & & Birth weight & \\
\hline Spontaneous & $289(79.0)$ & $<p 5$ & $125(33.4)$ \\
\hline$I V F$ & $7(1.9)$ & P5-p95 & $192(51.3)$ \\
\hline ICSI & $7(1.9)$ & $>p 95$ & $31(8.3)$ \\
\hline Other & $12(3.3)$ & Unknown & $26(7.0)$ \\
\hline Unknown & 51 (13.9) & & \\
\hline
\end{tabular}

532 Data are presented as number (percentage) or median and (range).

533

534 BMI Body Mass Index, IVF In vitro fertilization, ICSI Intracytoplasmic sperm injection.

535

536

537

538

539

540

541

542 


\section{Table 1. Rare CNVs in prenatal cohort.}

544

545

\begin{tabular}{lll} 
Copy Number Loss & & Number of cases \\
\hline Deleterious & $\geq 2 \mathrm{Mb \#}$ & 10 \\
Likely deleterious & $\geq 1 \mathrm{Mb}$ & 6 \\
VOUS & $\leq 1 \mathrm{Mb}$ inheritance unspecified & 20 \\
Likely Benign & present in DDD controls & 7 \\
\hline & & 43 \\
Copy Number Gain & & \\
\hline Deleterious & $\geq 3 \mathrm{Mb}$ or overlapping dominant disease & 4 \\
& genes & 3 \\
Likely deleterious & $\geq 2 \mathrm{Mb}$ & 17 \\
VOUS & $\leq 1 \mathrm{Mb}$ inheritance unspecified & 5 \\
Likely Benign & present in DDD controls & 29
\end{tabular}

$546 \quad$ \# The rare CNVs at $6 \mathrm{q} 27$ are classified as Deleterious.

547 DDD indicates Deciphering Developmental Disorders 